\title{
New improved massive gravity and three-dimensional spacetimes of constant curvature and constant torsion
}

\author{
Tekin Dereli $^{*}$ and Cem Yetişmişoğlu ${ }^{\dagger}$ \\ Department of Physics, Koç University, Sartyer, İstanbul 34450, Turkey
}

(Received 20 June 2016; published 23 September 2016)

\begin{abstract}
We derive the field equations for topologically massive gravity coupled with the most general quadratic curvature terms using the language of exterior differential forms and a first order constrained variational principle. We find variational field equations both in the presence and absence of torsion. We then show that spaces of constant negative curvature (i.e. the anti-de Sitter space $\mathrm{AdS}_{3}$ ) and constant torsion provide exact solutions.
\end{abstract}

DOI: 10.1103/PhysRevD.94.064067

\section{INTRODUCTION}

It is often useful to study lower dimensional field theoretic models to gain further insight into fundamental interactions of nature. In particular, gravity in $(1+2)$ dimensions has received a lot of attention as a theoretical tool that highlights the topological aspects of gravitation. Basic questions such as whether gravitational interactions may have a finite range [1] or in which sense a quantum gravity might be useful [2] have led to insightful answers with this approach. In fact it is well known that Einstein's gravity in $(1+2)$ dimensions has no dynamics on its own [3]. One may introduce gravitational degrees of freedom that propagate, either by elevating the gravitational field equations to third order by including in the action a topological Chern-Simons term [4,5], or by coupling other fields such as a dilaton scalar [6] or a gravitino field [7] to gravity. Topologically massive gravity (TMG) proved to be interesting since it admits a stationary, circularly symmetric BTZ solution that is asymptotically $\mathrm{AdS}_{3}$, and behaves as if it is a rotating black hole $[8,9]$. In many respects, the BTZ solution of TMG is the analog of the Kerr solution of general relativity in $(1+3)$ dimensions. The construction of conserved quantities associated with the BTZ solution $[10,11]$ and the study of hidden dualities $[12,13]$ prove to be challenging problems in their own right. More recently, unitary extensions of TMG were sought by the addition of quadratic curvature terms to the action, thus raising the order of the Einstein field equations from three in TMG to four [14-16]. A remarkable extension of TMG, that is called new massive gravity (NMG) in the literature $[17,18]$ consists of discarding the Chern-Simons term altogether in the action, so that there are no third derivatives left in the field equations, and replacing it by a particular combination of quadratic curvature invariants that leads to fourth order field equations, but with unitarity guaranteed at least at the linearized approximation. It should be noted that all the

\footnotetext{
*tdereli@ku.edu.edu

cyetismisoglu@ku.edu.tr
}

models of 3D gravity discussed up to this point involve (pseudo-)Riemannian spacetimes. A minimal massive gravity (MMG) was introduced a couple of years ago for which the variation of the action is done under a nonlinear constraint that induces a dynamical spacetime torsion [19-21]. Several aspects of MMG such as unitarity $[22,23]$ or its conserved quantities [24] and exact solutions $[25,26]$ have been the subject of very recent studies.

Here in what follows, the field equations for the EinsteinChern-Simons gravity coupled with the most general quadratic curvature terms in the action are derived by a first order constrained variational principle. We make extensive use of the concise language of exterior differential forms. Variational field equations both in the presence and absence of torsion are determined. The notion of the torsion of a material continuum was first introduced by Cartan in 1922 [27]. This idea found important physical applications, on the one hand, within the context of modified theories of gravity [28], while on the other hand within the context of gauge theories of continuum dislocation and disclination defects [29]. Here we will be discussing three-dimensional spacetimes of constant negative curvature (i.e. $\mathrm{AdS}_{3}$ ) and constant torsion [30,31] as exact background solutions. The concept of threedimensional spaces of constant torsion was implicit in Cartan's work and it is called Cartan's spiral staircase in a recent review paper [29].

\section{A. Notation and Conventions}

Throughout our work, we will be using the language of exterior differential forms [6,21]. The metric tensor of spacetime, given by $g=\eta_{a b} e^{a} \otimes e^{b}$ where $\eta_{a b}=$ $g\left(X_{a}, X_{b}\right)=\operatorname{diag}(-,+,+)$ is written in terms of an orthonormal basis of frame vectors $\left\{X_{a}\right\}$ that are dual to the coframe 1-forms $\left\{e^{a}\right\}$ so that $e^{a}\left(X_{b}\right)=\delta_{b}^{a} \cdot l_{a}=l_{X_{a}}$ stands for the interior product operators with respect to frame vectors $X_{a} . *: \Lambda^{p}(M) \rightarrow \Lambda^{3-p}(M)$, denotes the Hodge duality operator acting on $p$-forms. The spacetime orientation is fixed with the choice of the volume 3-form 
$* 1=e^{0} \wedge e^{1} \wedge e^{2}$. For convenience, the abbreviation for the exterior products $e^{a b \ldots}=e^{a} \wedge e^{b} \wedge \ldots$ is going to be used. A linear connection on spacetime will be specified by a set of connection 1-forms $\left\{\omega^{a}{ }_{b}\right\}$. We will work with a connection that is compatible with the metric but need not be torsion free. Then the index raising and lowering operations commute with the covariant exterior derivation and we have $D(\omega) \eta_{a b}=\omega_{a b}+\omega_{b a}=0$. We specify the torsion 2-forms $T^{a}$ of spacetime through the first set of Cartan structure equations

$$
d e^{a}+\omega^{a}{ }_{b} \wedge e^{b}=T^{a},
$$

while the curvature 2-forms $R^{a}{ }_{b}(\omega)$ through the second set of Cartan structure equations

$$
d \omega^{a}{ }_{b}+\omega^{a}{ }_{c} \wedge \omega_{b}^{c}=R_{b}^{a}(\omega) .
$$

The following Bianchi identities are obtained as integrability conditions of the above Cartan structure equations:

$$
D(\omega) T^{a}=R_{b}^{a}(\omega) \wedge e^{b}, \quad D(\omega) R^{a}{ }_{b}(\omega)=0 .
$$

It is convenient to define contortion 1 -forms $K^{a}{ }_{b}$ as the difference between our non-Riemannian connection 1-forms and the Riemannian (Levi-Cività) connection 1-forms $\left\{\hat{\omega}^{a}{ }_{b}\right\}$ that satisfy the structure equations

$$
d e^{a}+\hat{\omega}_{b}^{a} \wedge e^{b}=0
$$

Thus we have

$$
K^{a}{ }_{b}=\omega_{b}^{a}-\hat{\omega}^{a}{ }_{b}
$$

which are in one-to-one correspondence with the torsion 2-forms $T^{a}$ through the structure equations

$$
K^{a}{ }_{b} \wedge e^{b}=T^{a},
$$

or conversely

$$
2 K_{a b}=\iota_{a} T_{b}-\imath_{b} T_{a}-e^{c} \imath_{a b} T_{c} .
$$

It is not difficult to find a relation between the nonRiemannian curvature 2-forms $R^{a}{ }_{b}(\omega)$ and the Riemannian curvature 2-forms $R^{a}{ }_{b}(\hat{\omega})$ of the Levi-Cività connection as

$$
R_{b}^{a}(\omega)=R_{b}^{a}(\hat{\omega})+D(\hat{\omega}) K_{b}^{a}+K^{a}{ }_{c} \wedge K^{c}{ }_{b}
$$

where $D(\hat{\omega})$ denotes the covariant exterior derivative with respect to the Levi-Cività connection. The Ricci 1-forms $R i c_{a}=\mathcal{R}_{a b} e^{b}$ and the curvature scalar $\mathcal{R}$ are obtained by contractions of the curvature 2 -forms as follows:

$$
\text { Ric }_{a}=\iota^{b} R_{b a}, \quad \mathcal{R}=\imath^{a} \operatorname{Ric}_{a}=\iota^{a b} R_{b a} .
$$

Moreover, the Einstein 2-forms of our non-Riemannian connection are defined by

$G_{a}(\omega)=G_{a b} * e^{b}=* R i c_{a}-\frac{1}{2} \mathcal{R} * e_{a}=-\frac{1}{2} R^{b c}(\omega) * e_{a b c}$.

We note that in three dimensions, the curvature 2-forms are in one-to-one correspondence with the Einstein 2-forms since

$$
\epsilon_{a b c} G^{c}(\omega)=R_{a b}(\omega)
$$

where $\epsilon_{a b c}$ denotes the completely antisymmetric LeviCività symbol in three dimensions with $\epsilon_{012}=1$. We may therefore give the curvature 2-forms $R_{a b}$ in three dimensions in terms of the Ricci 1-forms $R i c_{a}$ and the curvature scalar $\mathcal{R}$ :

$$
R_{a b}=\epsilon^{c}{ }_{a b} * \operatorname{Ric}_{c}+\frac{1}{2} \mathcal{R} e_{a} \wedge e_{b}
$$

As a consequence, quadratic curvature invariants in three dimensions are related to each other through the identity

$$
R^{a b} \wedge * R_{a b}-2 \operatorname{Ric}^{a} \wedge * \operatorname{Ric}_{a}+\frac{1}{2} \mathcal{R}^{2} * 1=0
$$

that is, any one of the quadratic curvature invariants in three dimensions can be expressed in terms of the other two.

\section{ACTION}

The field equations of our model will be determined by the constrained variations of an action integral

$$
I\left[e^{a}, \omega^{a}{ }_{b}, \lambda_{a}\right]=\int_{M} \mathcal{L}
$$

where $M$ is a compact region on some chart on a $(1+2)$ dimensional Riemann-Cartan manifold. The independent variables on which the action depends are the coframe 1-forms $\left\{e^{a}\right\}$, connection 1-forms $\left\{\omega^{a}{ }_{b}\right\}$, and Lagrange multiplier 1-forms $\left\{\lambda_{a}\right\}$. We consider a Lagrangian density 3-form

$$
\mathcal{L}=\mathcal{L}_{\mathrm{TMG}}+\mathcal{L}_{2}+\mathcal{L}_{C}
$$

where

$$
\begin{aligned}
\mathcal{L}_{\mathrm{TMG}}= & \frac{1}{\mu}\left(\omega^{a}{ }_{b} \wedge d \omega^{b}{ }_{a}+\frac{2}{3} \omega^{a}{ }_{b} \wedge \omega^{b}{ }_{c} \wedge \omega^{c}{ }_{a}\right) \\
& +\frac{1}{2 K} R^{a b} \wedge * e_{a b}+\Lambda * 1
\end{aligned}
$$

is the Lagrangian density of the topologically massive gravity (TMG); 


$$
\mathcal{L}_{2}=\alpha R^{a b} \wedge * R_{a b}+\beta R i c^{a} \wedge * R i c_{a}+\gamma \mathcal{R}^{2} * 1
$$

is a generic quadratic curvature term with three coupling constants $\alpha, \beta$, and $\gamma$. It should be noted that there are alternative ways of specifying a generic quadratic curvature invariant in three dimensions. Due to the identity (1.13), any of the terms $R^{a b} \wedge * R_{a b}, R i c^{a} \wedge * \operatorname{Ric}_{a}$ or $\mathcal{R}^{2} * 1$ may be made redundant in favor of others. Therefore, still keeping the coupling constants $\alpha, \beta$ and $\gamma$, we may consider without loss of generality any one of the following combinations:

$$
\begin{aligned}
& \mathcal{L}_{2}=(2 \alpha+\beta) \operatorname{Ric}^{a} \wedge * \operatorname{Ric}_{a}+\left(\gamma-\frac{\alpha}{2}\right) \mathcal{R}^{2} * 1 \\
& \mathcal{L}_{2}=\left(\alpha+\frac{\beta}{2}\right) R^{a b} \wedge * R_{a b}+\left(\gamma+\frac{\beta}{4}\right) \mathcal{R}^{2} * 1 \\
& \mathcal{L}_{2}=(\alpha-2 \gamma) R^{a b} \wedge * R_{a b}+(\beta+4 \gamma) \operatorname{Ric}^{a} \wedge * \operatorname{Ric}_{a} .
\end{aligned}
$$

For technical ease. we prefer to work with the second alternative. Finally,

$$
\mathcal{L}_{C}=T^{a} \wedge \lambda_{a}+\frac{\nu}{2} \lambda_{a} \wedge \lambda_{b} \wedge * e^{a b}
$$

is the constraint Lagrangian density 3 -form, which in the case $\nu=0$ imposes the constraint that the connection is the torsion-free Levi-Cività connection. On the other hand if $\nu \neq 0$, the torsion 2-forms would be related with the Lagrange multiplier 1-forms in a nontrivial way. All the previously studied models such as TMG, NMG or MMG are covered as subcases with our choice of the action.

\section{VARIATIONAL FIELD EQUATIONS}

We evaluate the variational derivative of the total Lagrangian and find (up to a closed form)

$$
\begin{aligned}
\dot{\mathcal{L}}= & \dot{e}^{a} \wedge\left\{\frac{1}{2 K} R^{b c} \wedge * e_{a b c}+\Lambda * e_{a}+\left(\alpha+\frac{\beta}{2}\right)\left(l_{a} R^{b c} \wedge * R_{b c}-R^{b c} \wedge l_{a} * R_{b c}\right)+\left(\gamma+\frac{\beta}{4}\right)\left(2 \mathcal{R} R^{b c} \wedge * e_{a b c}-\mathcal{R}^{2} * e_{a}\right)\right. \\
& \left.+D(\omega) \lambda_{a}+\frac{\nu}{2} \lambda^{b} \wedge \lambda^{c} \wedge * e_{a b c}\right\}+\dot{\omega}^{a b} \wedge\left\{\frac{2}{\mu} R_{b a}+\frac{1}{2 K} T^{c} \wedge * e_{a b c}+(2 \alpha+\beta) D(\omega) * R_{a b}\right. \\
& \left.+\left(2 \gamma+\frac{\beta}{2}\right) D(\omega)\left(\mathcal{R} * e_{a b}\right)+\frac{1}{2}\left(e_{b} \wedge \lambda_{a}-e_{a} \wedge \lambda_{b}\right)\right\}+\dot{\lambda}_{a} \wedge\left\{T^{a}+\nu \lambda_{b} \wedge * e^{a b}\right\}
\end{aligned}
$$

Here a dot over a field variable denotes the variation of the corresponding field. We first impose the constraint

$$
T^{a}=-\nu \lambda_{b} \wedge * e^{a b} \Leftrightarrow K_{a b}=\nu \epsilon_{a b c} \lambda^{c} .
$$

Then we go to connection variation equations which now read

$$
e_{a} \wedge \lambda_{b}-e_{b} \wedge \lambda_{a}=Q^{-1} \Sigma_{a b}
$$

where we set

$$
Q=\frac{1}{2}-\frac{\nu}{2 K}-\nu\left(2 \gamma+\frac{\beta}{2}\right) \mathcal{R},
$$

and

$$
\begin{aligned}
\Sigma_{a b}= & -\frac{2}{\mu} R_{a b}+(2 \alpha+\beta) D(\omega) * R_{a b} \\
& +\left(2 \gamma+\frac{\beta}{2}\right) d \mathcal{R} \wedge * e_{a b} .
\end{aligned}
$$

We solve (3.3) algebraically for the Lagrange multiplier 1 -forms and determine

$$
\lambda_{a}=Q^{-1}\left(-\frac{2}{\mu} Y_{a}+(2 \alpha+\beta) W_{a}+\left(2 \gamma+\frac{\beta}{2}\right)\left(l_{a} * d \mathcal{R}\right)\right)
$$

where we introduced further abbreviations

$$
\begin{aligned}
Y_{a} & =\operatorname{Ric}_{a}-\frac{1}{4} e_{a} \mathcal{R}, \\
W_{a} & =l^{b}\left(D(\omega) * R_{b a}\right)-\frac{1}{4} e_{a}\left(l^{b} l^{c} D(\omega) * R_{c b}\right) .
\end{aligned}
$$

Finally we substitute (3.6) into the coframe variation equations and arrive at the Einstein field equations given as follows:

$$
\begin{aligned}
& \left(\frac{1}{2 K}+\left(2 \gamma+\frac{\beta}{2}\right) \mathcal{R}\right) R^{b c} \wedge * e_{a b c}+\left(\Lambda-\left(\gamma+\frac{\beta}{4}\right) \mathcal{R}^{2}\right) * e_{a} \\
& +\left(\alpha+\frac{\beta}{2}\right)\left(c_{a} R^{b c} \wedge * R_{b c}-R^{b c} \wedge l_{a} * R_{b c}\right) \\
& +D \lambda_{a}+\frac{\nu}{2} \lambda^{b} \wedge \lambda^{c} * e_{a b c}=0
\end{aligned}
$$

We note that these equations include among other terms, the Cotton-Schouten 2-forms 
TEKIN DERELI and CEM YETIŞMIŞOĞLU

$$
C_{a} \equiv D(\omega) Y_{a}=D(\omega)\left(\operatorname{Ric}_{a}-\frac{1}{4} \mathcal{R} e_{a}\right)
$$

that involve third derivatives of the metric components and the 2-forms

$$
\begin{aligned}
D_{a} & \equiv D(\omega) W_{a} \\
& =D(\omega)\left(\imath^{b}\left(D(\omega) * R_{b a}\right)-\frac{1}{4} e_{a} \iota^{b} \iota^{c}\left(D(\omega) * R_{c b}\right)\right)
\end{aligned}
$$

that involve fourth derivatives of the metric components. We also note that Einstein field equations in the case of zero torsion $(\nu=0)$ are given by

$$
\begin{aligned}
& \left(\frac{1}{2 K}+\left(2 \gamma+\frac{\beta}{2}\right) \hat{\mathcal{R}}\right) \hat{R}^{b c} \wedge * e_{a b c}+\left(\Lambda-\left(\gamma+\frac{\beta}{4}\right) \hat{\mathcal{R}}^{2}\right) * e_{a} \\
& +\left(\alpha+\frac{\beta}{2}\right)\left(\iota_{a} \hat{R}^{b c} \wedge * \hat{R}_{b c}-\hat{R}^{b c} \wedge l_{a} * \hat{R}_{b c}\right) \\
& -\frac{4}{\mu} \hat{C}_{a}+(4 \alpha+2 \beta) \hat{D}_{a}+(4 \gamma+\beta) D(\hat{\omega})\left(\iota_{a} * d \hat{\mathcal{R}}\right)=0 .
\end{aligned}
$$

These field equations go down consistently to the TMG field equations if the quadratic curvature terms are absent, i.e. if we set $\alpha=\beta=\gamma=0$ above.

Finally we rewrite the Einstein field equations in two special cases of recent interest.

New massive gravity: $\frac{1}{\mu} \rightarrow 0, \Lambda=0, \alpha=0, \beta=1, \gamma=-\frac{3}{8}$, $\nu=0$.

$$
\begin{gathered}
\left(\frac{1}{2 K}-\frac{1}{4} \hat{\mathcal{R}}\right) \hat{R}^{b c} \wedge * e_{a b c}+\frac{1}{8} \hat{\mathcal{R}}^{2} * e_{a} \\
+\frac{1}{2}\left(\iota_{a} \hat{R}^{b c} \wedge * \hat{R}_{b c}-\hat{R}^{b c} \wedge l_{a} * \hat{R}_{b c}\right) \\
+2 \hat{D}_{a}-\frac{1}{2} D(\hat{\omega})\left(\iota_{a} * d \hat{\mathcal{R}}\right)=0
\end{gathered}
$$

Minimal massive gravity: $\alpha=\beta=\gamma=0, \nu \neq 0$.

$$
\begin{aligned}
-\frac{1}{K} G_{a}+\Lambda * e_{a}= & \frac{4 K}{\mu(K-\nu)} D(\omega) Y_{a} \\
& -\frac{8 K^{2} \nu}{\mu^{2}(K-\nu)^{2}} Y^{b} \wedge Y^{c} \wedge * e_{a b c}=0 \\
K_{a b}= & -\frac{4 K \nu}{\mu(K-\nu)} \epsilon_{a b c} Y^{c}
\end{aligned}
$$

PHYSICAL REVIEW D 94, 064067 (2016)

\section{BACKGROUND SOLUTIONS WITH AdS}

In order to proceed any further in the study of a 3D quantized theory of gravity based on our model, its background solutions should be found. Towards that end, here we consider three-dimensional non-Riemannian spacetimes of constant curvature and constant torsion [30,31]. We also conveniently work with coordinate independent methods [6]. That is to say, we evaluate curvatures and their derivatives without differentiating any functions. The relevant differential geometric techniques are briefly explained in the Appendix. Our starting point will be the structure equations satisfied by an orthonormal set of leftinvariant basis 1 -forms $\left\{e^{a}\right\}$ on $\mathrm{AdS}_{3}$ :

$$
d e^{a}=-\frac{1}{\rho} \epsilon_{b c}^{a} e^{b} \wedge e^{c} .
$$

Thus we determine the Levi-Cività connection 1-forms

$$
\hat{\omega}^{a}{ }_{b}=-\frac{1}{\rho} \epsilon_{b c}^{a} e^{c},
$$

and the corresponding curvature 2-forms

$$
\hat{R}_{b}^{a}=-\frac{1}{\rho^{2}} e^{a} \wedge e_{b} .
$$

Now, we set the torsion 2-forms to be

$$
T^{a}=\frac{2}{\sigma} * e^{a}, \quad \sigma^{2} \neq \rho^{2} \Leftrightarrow K^{a}{ }_{b}=-\frac{1}{\sigma} \epsilon_{b c}^{a} e^{c} .
$$

Then the full curvature 2-forms turn out to be

$$
R_{b}^{a}=\left(\frac{\rho^{2}-\sigma^{2}}{\rho^{2} \sigma^{2}}\right) e^{a} \wedge e_{b}
$$

Their contractions give

$$
\text { Ric }_{a}=2\left(\frac{\rho^{2}-\sigma^{2}}{\rho^{2} \sigma^{2}}\right) e_{a}, \quad \mathcal{R}=6\left(\frac{\rho^{2}-\sigma^{2}}{\rho^{2} \sigma^{2}}\right) .
$$

Substituting these in (3.4), we find

$$
Q=\frac{1}{2}-\frac{\nu}{2 K}-6 \nu\left(2 \gamma+\frac{\beta}{2}\right)\left(\frac{\rho^{2}-\sigma^{2}}{\rho^{2} \sigma^{2}}\right),
$$

and in (3.6), we find

$$
\lambda_{a}=-Q^{-1}\left(\frac{1}{\mu}+\frac{2 \alpha+\beta}{\sigma}\right)\left(\frac{\rho^{2}-\sigma^{2}}{\rho^{2} \sigma^{2}}\right) e_{a} .
$$

We must first check (3.2) for consistency: 


$$
\frac{1}{\nu \sigma}=Q^{-1}\left(\frac{1}{\mu}+\frac{2 \alpha+\beta}{\sigma}\right)\left(\frac{\rho^{2}-\sigma^{2}}{\rho^{2} \sigma^{2}}\right) .
$$

Substituting for $Q$ from (4.7), we get an algebraic consistency equation as follows:

$$
2\left(\frac{\sigma}{\mu}+2 \alpha+4 \beta+12 \gamma\right)\left(\frac{\rho^{2}-\sigma^{2}}{\rho^{2} \sigma^{2}}\right)=\frac{K-\nu}{K \nu} .
$$

Next we go over to the Einstein field equations (3.8) with

$$
\lambda_{a}=-\frac{1}{\nu \sigma} e_{a}
$$

and organize terms to arrive at

$$
\begin{gathered}
(2 \alpha-2 \beta-12 \gamma)\left(\frac{\rho^{2}-\sigma^{2}}{\rho^{2} \sigma^{2}}\right)^{2}+\frac{1}{K}\left(\frac{\rho^{2}-\sigma^{2}}{\rho^{2} \sigma^{2}}\right) \\
+\left(\Lambda-\frac{1}{\nu \sigma^{2}}\right)=0 .
\end{gathered}
$$

Thus we obtain two algebraic equations (4.10) and (4.12) that are to be solved simultaneously for $\rho$ and $\sigma$ in terms of the coupling parameters $K, \Lambda, \alpha, \beta, \gamma, \mu$ and $\nu$. In order to make further headway, we introduce new variables

$$
\xi=\frac{1}{\rho}, \quad \eta=\frac{1}{\sigma}, \quad a=\frac{\mu}{4}\left(\frac{1}{\nu}-\frac{1}{K}\right)
$$

and write down the following simultaneous algebraic equations:

$$
\begin{aligned}
\frac{1}{(\xi+\eta)}+\frac{1}{(\xi-\eta)}= & \frac{1}{a^{2}}+\frac{(\alpha+2 \beta+6 \gamma)}{a}(\xi+\eta) \\
& +\frac{(\alpha+2 \beta+6 \gamma)}{a}(\xi-\eta),
\end{aligned}
$$

$$
\begin{aligned}
\left(\frac{\xi+\eta}{\xi-\eta}\right)+\left(\frac{\xi-\eta}{\xi+\eta}\right)= & \left(\frac{4 \nu}{K}-1\right)+\frac{4 \nu \Lambda}{(\xi-\eta)(\xi+\eta)} \\
& +4 \nu(2 \alpha-2 \beta-12 \gamma)(\xi-\eta)(\xi+\eta)
\end{aligned}
$$

Let us now make a further change of variables

$$
t=(\xi-\eta)+(\xi+\eta), \quad s=(\xi-\eta)(\xi+\eta),
$$

in terms of which we have

$$
a t=\frac{1}{a} s+(\alpha+2 \beta+6 \gamma) t s,
$$

$t^{2}=\left(1+\frac{4 \nu}{K}\right) s+4 \nu(2 \alpha-2 \beta-12 \gamma) s^{2}+4 \nu \Lambda$.

It is possible now to solve the first equation for $t$ in terms of $s$ and substitute in the second equation. Thus a quartic equation for $s$ is reached whose solutions can be obtained in a standard way. Then working through the equations backwards, solutions for $\rho$ and $\sigma$ may be written explicitly. As they stand, they are not very instructive. However, to be concrete, we concentrate on a simpler case and discuss the parameter ranges for the existence of solutions for MMG with $\alpha=\beta=\gamma=0$, in which case our coupled system of algebraic equations reduces to the equations of two conic sections in the $(\xi \eta)$-plane, given by

$$
\frac{(\xi-a)^{2}}{a^{2}}-\frac{\eta^{2}}{a^{2}}=1
$$

and

$$
K\left(\frac{1}{\nu}-\frac{1}{K}\right) \frac{\xi^{2}}{K \Lambda}+\frac{\eta^{2}}{K \Lambda}=1 .
$$

We take $K>0$ without loss of generality at this point, since our model is not yet coupled to matter. We also point out that solutions come in pairs with values $\eta \leftrightarrow-\eta$, as a change in sign of $\eta$ means going from one orientation of the cobasis to the other, or vice versa. In what follows, we restrict attention to the cases $0<\eta$, but extension to cases $\eta<0$ is easy. Then we classify possible pairs $(\xi, \eta)$ in accordance with the following ranges of our free parameters:

(i) For $\Lambda<0$ and $-\infty<\mu<\infty$; no solution exists with $0 \leq \nu \leq K$.

(ii) $\Lambda<0, \mu>0, \nu \leq 0$ or $K \leq \nu$.

There is a solution for $0 \leq \xi \leq \sqrt{\left|\frac{(K-\nu)}{\nu} K \Lambda\right|}$ and $0<\eta<\infty$. A second one may exist for $-\sqrt{\left|\frac{(K-\nu)}{\nu} K \Lambda\right|} \leq \xi \leq-\mu\left|\frac{(K-\nu)}{2 K \nu}\right|$, depending on the magnitude of $\mu$.

(iii) $\Lambda<0, \mu<0, \nu \leq 0$ or $K \leq \nu$.

There is a solution for $\xi \leq-\sqrt{\left|\frac{(K-\nu)}{\nu} K \Lambda\right|}$ and $0<\eta<\infty$. A second one may exist for $\sqrt{\left|\frac{(K-\nu)}{\nu} K \Lambda\right|} \leq \xi \leq \mu \frac{(K-\nu)}{2 K \nu}$, depending on the magnitude of $\mu$.

(iv) $\Lambda>0, \mu>0,0 \leq \nu \leq K$.

Solutions exist for $0 \leq \eta \leq \sqrt{K \Lambda}$. Then there is a solution for $-\sqrt{\frac{K-\nu}{\nu} K \Lambda} \leq \xi \leq 0$. A second one may exist, depending on the magnitude of $\mu$, for $\sqrt{\frac{K-\nu}{\nu} K \Lambda} \leq \xi \leq \mu \frac{(K-\nu)}{2 K \nu}$. 
(v) $\Lambda>0, \mu<0, \nu \leq 0$ or $K \leq \nu$.

Two solutions exist for $\sqrt{K \Lambda} \leq \eta$ and with either $\xi \leq 0$ or $\mu \frac{(K-\nu)}{2 K \nu} \leq \xi$.

(vi) $\Lambda>0, \mu>0, \nu \leq 0$ or $K \leq \nu$.

Two solutions exist for $\sqrt{K \Lambda} \leq \eta$ and with either $\xi \leq-\mu \frac{(K-\nu)}{2 K \nu}$ or $0 \leq \xi$.

(vii) $\Lambda>0, \mu<0,0 \leq \nu \leq K$.

Solutions exist for $\sqrt{K \Lambda} \leq \eta$. One solution has $0 \leq \xi \leq \sqrt{\frac{\nu}{K-\nu} K \Lambda}$. A second one may exist, depending on the magnitude of $\mu$, if $-\sqrt{\frac{\nu}{K-\nu} K \Lambda} \leq \xi \leq-\sqrt{|\mu| \frac{(K-\nu)}{\nu}}$.

\section{CONCLUSION}

In this paper, we considered the extension of EinsteinChern-Simons gravity (TMG) with the most general quadratic curvature terms in the action and derived the corresponding field equations by a first order constrained variational principle. Field equations were determined both in the absence and presence of a dynamical spacetime torsion. It should be emphasized that the discussion based on our choice of the action encompasses all currently studied models such as NMG or MMG as particular subcases. In order to specify the ground state of our model we then considered Riemann-Cartan spacetimes of constant negative curvature (i.e. $\mathrm{AdS}_{3}$ ) and constant torsion as exact background solutions. We conclude with the following comments:

(1) The main interest in models such as those considered here comes from the so-called unitarity clash problem between unitarity of the theory in the bulk and their dual on the boundary. The possibility to have dynamical torsion at the same time is also interesting. Even if both these goals can be achieved in the absence of quadratic curvature terms in the action, as is well known, any such modification can easily breaks the unitarity of the theory leading to the appearance of ghosts. The concise expressions we derive are geometrically transparent and easier to analyze.

(2) The generic quadratic curvature term in the action that is commonly used in recent literature is given by the first alternative in (2.5). Here we use the second alternative for technical ease and were able to present the final field equations (3.8) in a much more concise way.

(3) For both TMG and NMG (as well as MMG) it is already known that by the introduction of one or two auxiliary 1-forms one can rewrite such theories in the Chern-Simons-like form, thus avoiding the usage of the Hodge * operator and making it more convenient to investigate the presence or absence of ghosts. In [19], such a formulation is explicitly given but for the case of zero torsion only. The more general model considered here may also be reformulated in such a convenient form and that is worth exploring in a separate paper.

(4) The notion of three-dimensional Riemann-Cartan spaces with constant curvature and constant torsion is not new [30,31], but has not been used in this context before. It provides a novel pathway for the construction of physically relevant 3D-gravity configurations.

\section{ACKNOWLEDGMENTS}

C. Y. thanks Koç University for a Graduate Student Scholarship.

\section{APPENDIX: GEOMETRY OF AdS 3}

$\mathrm{AdS}_{3}$ can be realized as an embedded hypersurface in a four-dimensional flat space equipped with an indefinite metric $g=-d U^{2}-d V^{2}+d X^{2}+d Y^{2}$, written in Cartesian coordinates $\left\{\xi^{A} ; A=1,2,3,4\right\}:(U, V, X, Y)$. In the same coordinate system, the embedding equation will be given by

$$
-U^{2}-V^{2}+X^{2}+Y^{2}=-1 .
$$

Furthermore we know that $\mathrm{AdS}_{3}$ is a homogeneous space with $\mathrm{AdS}_{3}=S O(2,2) / S O(2,1)$. In order to verify that the Lie algebra $s o(2,2)$ of isometries is a direct product of two copies of $s o(2,1)$, we consider, in our Cartesian system $\xi_{A}:(-U,-V, X, Y)$, the Killing vector fields $J_{A B}$ that are given explicitly by

$$
J_{A B}=\xi_{A} \frac{\partial}{\partial \xi^{B}}-\xi_{B} \frac{\partial}{\partial \xi^{A}}
$$

and satisfy the commutation relations:

$$
\left[J_{A B}, J_{B C}\right]= \begin{cases}-J_{A C}, & \text { for } B \in\{1,2\} \text { and } A \neq B \neq C \\ J_{A C}, & \text { for } B \in\{3,4\} \text { and } A \neq B \neq C\end{cases}
$$

It is straightforward to divide these Killing vector fields into two conjugacy classes by defining the left-invariant vector fields

$X_{0}=-J_{U V}-J_{X Y}, \quad X_{1}=J_{X U}+J_{Y V}, \quad X_{2}=J_{Y U}-J_{X V}$,

and the right-invariant vector fields

$Y_{0}=-J_{U V}+J_{X Y}, \quad Y_{1}=J_{X U}-J_{Y V}, \quad Y_{2}=-J_{Y U}-J_{X V}$. 
Both the left-invariant vector fields $\left\{X_{a}: a=0,1,2\right\}$ and the right-invariant vector fields $\left\{Y_{a}: a=0,1,2\right\}$ satisfy the same commutation relations

$$
\left[X_{0}, X_{1}\right]=2 X_{2}, \quad\left[X_{1}, X_{2}\right]=-2 X_{0}, \quad\left[X_{0}, X_{2}\right]=-2 X_{1},
$$

$\left[Y_{0}, Y_{1}\right]=2 Y_{2}, \quad\left[Y_{1}, Y_{2}\right]=-2 Y_{0}, \quad\left[Y_{0}, Y_{2}\right]=-2 Y_{1}$,

and they commute with each other, i.e.

$$
\left[X_{a}, Y_{b}\right]=0, \quad a, b=0,1,2 .
$$

At this point, we choose a local coordinate chart $x^{\mu}:(t, \chi, \theta)$ for $\mathrm{AdS}_{3}$ such that

$$
\begin{aligned}
& U=\cos t, \quad V=\sin t \cosh \chi, \\
& X=\sin t \sinh \chi \cos \theta, \quad Y=\sin t \sinh \chi \sin \theta .
\end{aligned}
$$

Then we compute the following explicit expressions for $\left\{X_{a}\right\}$,

$$
\begin{aligned}
X_{0}= & \cosh \chi \partial_{t}-\cot t \sinh \chi \partial_{\chi}-\partial_{\theta}, \\
X_{1}= & -\sinh \chi \cos \theta \partial_{t}+(\cot t \cosh \chi \cos \theta+\sin \theta) \partial_{\chi} \\
& +(\operatorname{coth} \chi \cos \theta-\cot t \operatorname{cosech} \chi \sin \theta) \partial_{\theta}, \\
X_{2}= & -\sinh \chi \sin \theta \partial_{t}+(\cot t \cosh \chi \sin \theta-\cos \theta) \partial_{\chi} \\
& +(\operatorname{coth} \chi \sin \theta+\cot t \operatorname{cosech} \chi \cos \theta) \partial_{\theta},
\end{aligned}
$$

and for $\left\{Y_{a}\right\}$,

$$
\begin{aligned}
Y_{0}= & \cosh \chi \partial_{t}-\cot t \sinh \chi \partial_{\chi}+\partial_{\theta}, \\
Y_{1}= & -\sinh \chi \cos \theta \partial_{t}+(\cot t \cosh \chi \cos \theta-\sin \theta) \partial_{\chi} \\
& +(-\operatorname{coth} \chi \cos \theta-\cot t \operatorname{cosech} \chi \sin \theta) \partial_{\theta}, \\
Y_{2}= & \sinh \chi \sin \theta \partial_{t}+(-\cot t \cosh \chi \sin \theta-\cos \theta) \partial_{\chi} \\
& +(\operatorname{coth} \chi \sin \theta-\cot t \operatorname{cosech} \chi \cos \theta) \partial_{\theta} .
\end{aligned}
$$

Finally, exploiting the dualities $e^{b}\left(X_{a}\right)=\delta_{a}^{b}$ and $\tilde{e}^{b}\left(Y_{a}\right)=$ $\delta_{a}^{b}$, we determine in a unique way the following set of leftinvariant coframe 1 -forms,

$$
\begin{aligned}
e^{0}= & \cosh \chi d t+\cos t \sin t \sinh \chi d \chi+\sin ^{2} t \sinh ^{2} \chi d \theta, \\
e^{1}= & \sinh \chi \cos \theta d t+\left(\cos t \sin t \cosh \chi \cos \theta+\sin ^{2} t \sin \theta\right) d \chi \\
& +\sin ^{2} t \sinh \chi(\cosh \chi \cos \theta-\cot t \sin \theta) d \theta, \\
e^{2}= & \sinh \chi \sin \theta d t+\left(\cos t \sin t \cosh \chi \sin \theta-\sin ^{2} t \cos \theta\right) d \chi \\
& +\sin ^{2} t \sinh \chi(\cosh \chi \sin \theta+\cot t \cos \theta) d \theta,
\end{aligned}
$$

and the right-invariant coframe 1-forms,

$\tilde{e}^{0}=\cosh \chi d t+\cos t \sin t \sinh \chi d \chi-\sin ^{2} t \sinh ^{2} \chi d \theta$,

$\tilde{e}^{1}=\sinh \chi \cos \theta d t+\left(\cos t \sin t \cosh \chi \cos \theta-\sin ^{2} t \sin \theta\right) d \chi$

$-\sin ^{2} t \sinh \chi(\cosh \chi \cos \theta+\cot t \sin \theta) d \theta$,

$\tilde{e}^{2}=-\sinh \chi \sin \theta d t-\left(\cos t \sin t \cosh \chi \sin \theta+\sin ^{2} t \cos \theta\right) d \chi$

$+\sin ^{2} t \sinh \chi(\cosh \chi \sin \theta-\cot t \cos \theta) d \theta$.

It is now straightforward to verify (i) that these basis 1 -forms satisfy the first Cartan structure equations

$$
\begin{aligned}
d e^{a} & =-\epsilon^{a}{ }_{b c} e^{b} \wedge e^{c}, \\
d \tilde{e}^{a} & =-\epsilon^{a}{ }_{b c} \tilde{e}^{b} \wedge \tilde{e}^{c},
\end{aligned}
$$

and that (ii) in our local coordinate chart the metric tensor becomes

$$
\begin{aligned}
g_{\mathrm{AdS}_{3}} & =-e^{0} \otimes e^{0}+e^{1} \otimes e^{1}+e^{2} \otimes e^{2} \\
& =-\tilde{e}^{0} \otimes \tilde{e}^{0}+\tilde{e}^{1} \otimes \tilde{e}^{1}+\tilde{e}^{2} \otimes \tilde{e}^{2} \\
& =-d t^{2}+\sin ^{2} t\left(d \chi^{2}+\sinh ^{2} \chi d \theta^{2}\right) .
\end{aligned}
$$

As a further remark, suppose we let the right-invariant vector fields change sign i.e. $Y_{a} \mapsto W_{a}=-Y_{a}, a=0,1,2$, so that the volume form also switches sign. Then the corresponding basis 1 -forms $\left\{\bar{e}^{a}\right\}$ differ from the rightinvariant 1 -forms $\left\{\tilde{e}^{a}\right\}$ by an overall minus sign,

$\bar{e}^{0}=-\cosh \chi d t-\cos t \sin t \sinh \chi d \chi+\sin ^{2} t \sinh ^{2} \chi d \theta$,

$\bar{e}^{1}=-\sinh \chi \cos \theta d t+(-\cos t \sin t \cosh \chi \cos \theta$

$\left.+\sin ^{2} t \sin \theta\right) d \chi+\sin ^{2} t \sinh \chi(\cosh \chi \cos \theta$

$+\cot t \sin \theta) d \theta$,

$\bar{e}^{2}=\sinh \chi \sin \theta d t+(\cos t \sin t \cosh \chi \sin \theta$

$\left.+\sin ^{2} t \cos \theta\right) d \chi-\sin ^{2} t \sinh \chi(\cosh \chi \sin \theta$

$-\cot t \cos \theta) d \theta$,

and satisfy the following structure equations:

$$
d \bar{e}^{a}=\epsilon_{b c}^{a} \bar{e}^{b} \wedge \bar{e}^{c} .
$$


[1] D. Boulware and S. Deser, Can gravitation have a finite range?, Phys. Rev. D 6, 3368 (1972).

[2] H. Leutwyler, A $2+1$ dimensional model for the quantum theory of gravity, Nuovo Cimento A 42, 159 (1966).

[3] S. Deser, R. Jackiw, and G. 't Hooft, Three dimensional Einstein gravity: Dynamics of flat space, Ann. Phys. (N.Y.) 152, 220 (1984); Three-dimensional cosmological gravity: Dynamics of constant curvatureAnn. Phys. (N.Y.) 153, 405 (1984).

[4] S. Deser, R. Jackiw, and S. Templeton, Topologically Massive Gauge Theories, Phys. Rev. Lett. 48, 975 (1982).

[5] S. Deser, R. Jackiw, and S. Templeton, Topologically massive gauge theories, Ann. Phys. (N.Y.) 140, 372 (1982); 185, 406(E) (1988).

[6] T. Dereli and R. W. Tucker, Gravitational interactions in 2+1 dimensions, Classical Quantum Gravity 5, 951 (1988).

[7] T. Dereli and S. Deser, Fermionic Goldstone-Higgs effect in $2+1$ dimensions, J. Phys. A 11, L27 (1978).

[8] M. Banados, C. Teitelboim, and J. Zanelli, The Black Hole in Three Dimensional Space-Time, Phys. Rev. Lett. 69, 1849 (1992).

[9] M. Banados, M. Henneaux, C. Teitelboim, and J. Zanelli, Geometry of the $2+1$ black hole, Phys. Rev. D 48, 1506 (1993).

[10] J. D. Brown and M. Henneaux, Central charges in the canonical realization of asymptotical symmetries: An example from three dimensional gravity, Commun. Math. Phys. 104, 207 (1986).

[11] S. Deser and B. Tekin, Energy in topologically massive gravity, Classical Quantum Gravity 20, L259 (2003).

[12] T. Dereli and Yu. N. Obukhov, General analysis of self-dual solutions of Einstein-Maxwell-Chern-Simons theory in $(1+2)$ dimensions, Phys. Rev. D 62, 024013 (2000).

[13] S. Carlip, S. Deser, A. Waldron, and D. K. Wise, Topologically massive AdS gravity, Phys. Lett. B 666, 272 (2008).

[14] S. Deser, Ghost-Free, Finite 4th Order $D=3$ Gravity, Phys. Rev. Lett. 103, 101302 (2009).

[15] W. Li, W. Song, and A. Strominger, Chiral gravity in three dimensions, J. High Energy Phys. 04 (2008) 082.

[16] M. Nakasone and I. Oda, On unitarity of massive gravity in three dimensions, Prog. Theor. Phys. 121, 1389 (2009).
[17] E. A. Bergshoeff, O. Hohm, and P. K. Townsend, Massive Gravity in Three Dimensions, Phys. Rev. Lett. 102, 201301 (2009).

[18] E. A. Bergshoeff, O. Hohm, and P. K. Townsend, More on massive 3D gravity, Phys. Rev. D 79, 124042 (2009).

[19] H. R. Afshar, E. A. Bergshoeff, and W. Merbis, Extended massive gravity in three dimensions, J. High Energy Phys. 08 (2014) 115.

[20] E. A. Bergshoeff, O. Hohm, W. Merbis, A. J. Routh, and P. K. Townsend, Minimal massive 3D gravity, Classical Quantum Gravity 31, 145008 (2014).

[21] A. Baykal, An alternative derivation of the minimal massive 3D gravity, Classical Quantum Gravity 32, 025013 (2015).

[22] B. Tekin, Minimal massive gravity: Conserved charges, excitations, and the chiral gravity limit, Phys. Rev. D 90, 081701(R) (2014).

[23] M. Alishahiha, M. M. Qaemaqami, A. Haseh, and A. Shirzadi, On 3D minimal massive gravity, J. High Energy Phys. 12 (2014) 033.

[24] E. Altas and B. Tekin, Holographically viable extensions of topologically massive and minimal massive gravity, Phys. Rev. D 93, 025033 (2016).

[25] A.S. Arvanitakis, On solutions of minimal massive 3D gravity, Classical Quantum Gravity 32, 115010 (2015).

[26] E. Altas and B. Tekin, Exact solutions and the consistency of 3D minimal massive gravity, Phys. Rev. D 92, 025033 (2015).

[27] É. Cartan, Sur une généralisation de la notion de courbure de Riemann et les espacesà torsion, C.R. Hebd. Seances Acad. Sci. 174, 593 (1922).

[28] A.Trautman, Einstein-Cartan theory, in Encyclopedia of Mathematical Physics, edited by J.-P. Françoise, G. L. Naber, and S. T. Tsou (Elsevier, New York, 2006), Vol. 2, p. 189.

[29] M. Lazar and F. W. Hehl, Cartan's spiral staircase in physics, and in particular, in the gauge theory of dislocations, Found. Phys. 40, 1298 (2010).

[30] T. Dereli and A. Verçin, A gauge model of amorphous solids containing defects, Philos. Mag. B 56, 625 (1987).

[31] T. Dereli and A. Verçin, A gauge model of amorphous solids containing defects II. Chern-Simons free energy, Philos. Mag. B 64, 509 (1991). 